Case Reports

\title{
Emphysematous Pyelonephritis with Abscesses Masquerading as an Ordinary Urinary Tract Infection
}

\author{
${ }^{1}$ Tasnim F. Imran, ${ }^{1}$ Frederick Yick, ${ }^{2}$ Ahmed H. Qavi, ${ }^{1}$ Sundar Dhamotharan and ${ }^{1,3}$ Steven J. Sperber \\ ${ }^{I}$ Department of Medicine, New Jersey Medical School Rutgers, \\ The State University of New Jersey, 185 South Orange Avenue Newark, NJ 07103, United States \\ ${ }^{2}$ Department of Medicine, Dartmouth Hitchcock Medical Center Geisel, School of Medicine One Medical Center Drive \\ Lebanon, NH, 03756, United States \\ ${ }^{3}$ Division of Infectious Diseases Hackensack, University Medical Center 30 Prospect Ave Hackensack, NJ 07601 United States
}

\author{
Article history \\ Received: 16-03-2015 \\ Revised: 1-08-2015 \\ Accepted: 15-10-2015 \\ Corresponding Author: \\ Tasnim F. Imran \\ Department of Medicine, New \\ Jersey Medical School Rutgers, \\ The State University of New \\ Jersey 185 South Orange \\ Avenue Newark, NJ 07103, \\ United States \\ E-mail: imranta@njms.rutgers.edu
}

\begin{abstract}
Emphysematous Pyelonephritis (EPN) is a rare, gas-forming infection of the renal parenchyma associated with significant morbidity and mortality. We report the case of a 39 year-old woman with uncontrolled diabetes who presented with abdominal pain, fevers and chills for one month. Physical exam was notable for dry oral mucosa and left costovertebral angle tenderness. Labs showed acute kidney injury and a hyperglycemic hyperosmotic state. CT abdomen revealed emphysematous pyelonephritis with a large left renal abscess. EPN should be considered in diabetic patients, especially those with uncontrolled or new-onset diabetes and those presenting with symptoms of urinary tract obstruction. Prognostic factors include thrombocytopenia, shock, altered mental status and need for hemodialysis. Our patient was treated successfully with intravenous antibiotics, percutaneous drainage, strict glucose control and monitored with serial imaging. She underwent laparoscopic nephrectomy one month later. Early diagnosis of EPN is essential as it carries a high mortality and a strong clinical suspicion is required, as patients may have an atypical presentation.
\end{abstract}

Keywords: Emphysematous, Pyelonephritis, Diabetes Mellitus, Complicated Urinary Tract Infections

\section{Introduction}

Emphysematous Pyelonephritis (EPN) is a rare necrotizing infection of the renal parenchyma, perinephric tissue and surrounding areas caused by gas forming organisms. One of the first accounts of gas forming kidney infection was described by Kelly and MacCallum in 1898. EPN falls under a spectrum of gas forming infections of the urinary tract that include emphysematous pyelitis and cystitis. The mean patient age is approximately 60 years and there is female predominance, with a ratio of 6:1. Diabetes was noted in about $90 \%$ of patients and urinary tract obstruction in approximately 20\% (Aswathaman et al., 2008; Best et al., 1999). Although these infections account for only a small percentage of all urinary tract infections, recognition and early diagnosis is essential as they are associated with a high morbidity and mortality (Yao et al., 2007). Pyelitis must be differentiated from pyelonephritis, as the former is often treated successfully with antibiotics alone, while the latter can be life threatening. We describe a case of a patient who had an atypical presentation of emphysematous pyelonephritis and was treated early with intravenous antibiotics, percutaneous drainage and strict glucose control.

\section{Case Presentation}

A 39-year-old woman with newly diagnosed diabetes presented with nausea and abdominal pain for three days. The pain was diffuse, dull, and not related to eating. Associated symptoms included malaise and fatigue with exertion. Upon further questioning, the patient also reported fever, chills and polyuria over the past month. She denied dysuria, diarrhea, chest pain, shortness of breath, or lower extremity edema. She worked as a nurse practitioner in a nearby nursing home. She was diagnosed with diabetes about a month ago, treated with Glimiperide and has a history of iron deficiency anemia secondary to menorrhagia. 
On initial evaluation, the patient was noted to be febrile to $103 \mathrm{~F}$, with tachycardia to 144 beats $/ \mathrm{min}$, with a blood pressure of $92 / 59 \mathrm{mmHg}$, respiratory rate of 22 breaths/min and an oxygen saturation of $96 \%$ on ambient air. Her physical exam was remarkable for dry oral mucous membranes and non-specific left lower back tenderness, with a soft abdomen, non-tender, non-distended. Initial laboratory values were significant for acute kidney injury, hyperglycemia, hyponatremia, leukocytosis with neutrophilic predominance, thrombocytopenia and a microcytic anemia. Urinalysis showed cloudy urine, specific gravity of 1.020, glycosuria and 20-30 WBCs. Table 1 lists pertinent laboratory tests and their results.

A renal ultrasound was obtained to rule out hydronephrosis, which showed a complex mass on the lower pole of the left kidney and pyelocalciectasis. A CT scan of the abdomen and pelvis revealed emphysematous pyelonephritis with gas extending through the medulla into the cortex in the upper pole of the left kidney and a $6 \mathrm{~cm}$ fluid collection in the lower pole (Fig. 1). The patient underwent emergent drainage of the abscess of the left kidney with a placement of a percutaneous drain. About $75 \mathrm{cc}$ of purulent drainage was removed initially but the patient continued to have purulent drainage for several days after the procedure. Blood culture, urine culture and culture from the purulent drainage all grew Klebsiella pneumoniae. The patient was initially treated with piperacillin-tazobactam as the bacteria was found to be sensitive with a minimum inhibitory concentration of less than or equal to 4 . However, she continued to have fevers and leukocytosis with bandemia. A repeat CT scan of the abdomen and pelvis done ten days into the admission showed a new medially located left perinephric abscess in the midpole region (Fig. 1). This new abscess was drained and a second percutaneous drain was placed. Again purulent drainage was noted for a few days after placement of the second percutaneous drain. On day 11 of antibiotic treatment with piperacillin-tazobactam due to continued fevers and leukocytosis, the antibiotic regimen was changed to Meropenem. The second abscess cultures also grew Klebsiella pneumonia. The patient underwent a renal scan which revealed $27 \%$ function of the left kidney.

A month later the patient was readmitted with worsening renal function and the decision was made to proceed with nephrectomy. The patient underwent laporascopic left radical nephrectomy and was continued on intravenous Meropenem for three days after the procedure and then switched to Cefazolin for three days. She was discharged on oral Cephalexin for one week. Our patient was successfully treated with antibiotics, percutaneous drainage, tight glucose control, monitoring with serial imaging and ultimately required nephrectomy.

Table 1. Laboratory values

\begin{tabular}{|c|c|c|}
\hline Serum/blood lab test & Results & Reference range \\
\hline Sodium & $125 \mathrm{mmolL}$ & $136-142 \mathrm{mmol} / \mathrm{l}$ \\
\hline Potassium & $4.2 \mathrm{mmol} / 1$ & $3.5-5.0 \mathrm{mmol} / 1$ \\
\hline Chloride & $86 \mathrm{mmol} / \mathrm{l}$ & $98-108 \mathrm{mmol} / 1$ \\
\hline Bicarbonate & $21 \mathrm{mmol} / 1$ & $23-32 \mathrm{mmol} / \mathrm{l}$ \\
\hline Urea nitrogen & $46 \mathrm{mg} \mathrm{dL}^{-1}$ & $9-25 \mathrm{mg} \mathrm{dL}^{-1}$ \\
\hline Creatinine & $2.0 \mathrm{mg} \mathrm{dL}^{-1}$ & $0.5-1.1 \mathrm{mg} \mathrm{dL}^{-1}$ \\
\hline Glucose & $536 \mathrm{mg} \mathrm{dL}^{-1}$ & $54-118 \mathrm{mg} \mathrm{dL}^{-1}$ \\
\hline Protein & $6.3 \mathrm{~g} \mathrm{dL}^{-1}$ & $6.4-8.3 \mathrm{~g} \mathrm{dL}^{-1}$ \\
\hline Albumin & $3.1 \mathrm{~g} \mathrm{dL}^{-1}$ & $3.7-5.4 \mathrm{~g} \mathrm{dL}^{-1}$ \\
\hline Total bilirubin & $0.8 \mathrm{mg} /$ & $0.0-1.0 \mathrm{mg} \mathrm{dL}^{-1}$ \\
\hline AST & $75 \mathrm{U} / 1$ & $7-30 \mathrm{U} / 1$ \\
\hline ALT & $51 \mathrm{U} / 1$ & $9-32 \mathrm{U} / 1$ \\
\hline Alkaline Phosphatase & $146 \mathrm{U} / 1$ & $30-100 \mathrm{U} / 1$ \\
\hline Lactic Acid & $3.4 \mathrm{mmol} / 1$ & $0.5-2.2 \mathrm{mmol} / 1$ \\
\hline Acetone & Negative & Negative \\
\hline Hemoglobin A1C & 13.1 & $3.8-6.4$ \\
\hline \multicolumn{3}{|l|}{$C B C$} \\
\hline White blood count & $19.8 \mathrm{k} / \mathrm{mcl}$ & $4.5-11.0 \mathrm{k} / \mathrm{mcl}$ \\
\hline Neutrophils & $92 \%$ & $40-70 \%$ \\
\hline Lymphocytes & $4 \%$ & $22-44 \%$ \\
\hline Monocytes & $2 \%$ & $0-10 \%$ \\
\hline Eosinophils & $1 \%$ & $0-3 \%$ \\
\hline Basophils & $1 \%$ & $0-1 \%$ \\
\hline Hemoglobin & $9.0 \mathrm{~g} \mathrm{dL}^{-1}$ & $11.7-16.0 \mathrm{~g} / \mathrm{dl}$ (women) \\
\hline Hematocrit & $27.90 \%$ & $35-45 \%$ (women) \\
\hline Platelets & $103 \mathrm{k} / \mathrm{mcl}$ & $150-350 \mathrm{k} / \mathrm{mcl}$ \\
\hline $\mathrm{MCV}$ & $79.0 \mathrm{fl}$ & $80-100 \mathrm{fl}$ \\
\hline \multicolumn{3}{|l|}{ Urine } \\
\hline Urinalysis & $\begin{array}{l}\text { Cloudy, pH 5.5, specific gravity } 1.020 \text {, glucose } 500 \mathrm{mg} / \mathrm{dL} \text {, moderate blood, } \\
4+\text { protein, 20-30 WBCs, many bacteria, } 8-12 \text { squamous epithelial cells }\end{array}$ & \\
\hline Beta HCG & Negative & Negative \\
\hline
\end{tabular}



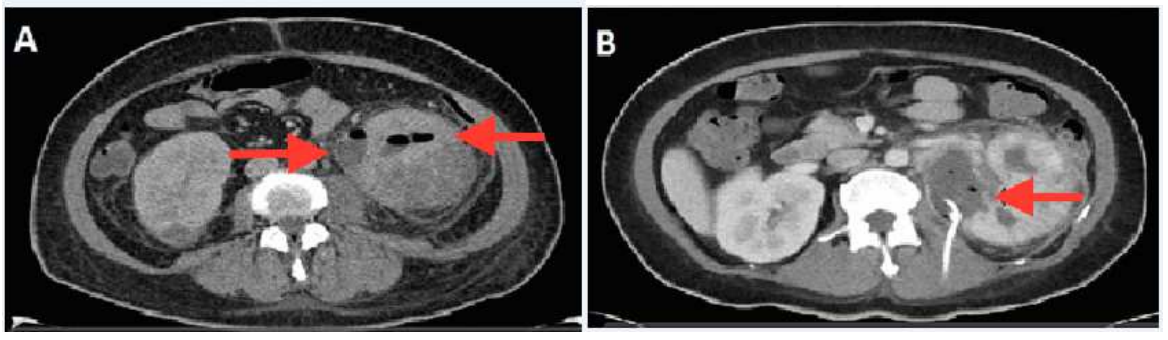

Fig. 1. (A) Computed tomography scan of the abdomen revealed left emphysematous pyelonephritis-air radiating through the medulla into the cortex (arrow, right), a complex fluid collection in the inferior pole measuring $6.2 \times 6.4 \mathrm{~cm}$ (arrow, left) and air tracking into the retroperitoneum (B) Repeat CT abdomen 10 days after treatment with intravenous antibiotics and percutaneous drainage demonstrated an enlarged left kidney with areas of necrosis and a decrease in abscess size and amount of air (arrow)

\section{Discussion}

Major risk factors for emphysematous pyelonephritis include uncontrolled diabetes mellitus and urinary tract obstruction. These infections occur with a higher frequency in females and may be due to the higher occurrence of urinary tract infections in females. A few cases have been reported in non-diabetic patients with a history of immunosuppression or urinary tract obstruction secondary to nephrolithiasis or a tumor (Ali and Barlas, 2010; Mokabberi and Ravakhah, 2007). Patients with uncontrolled diabetes, such as our patient, are particularly susceptible to these infections due to high levels of glycosylated hemoglobin and impaired host defense mechanisms.

Factors that may be involved in the pathogenesis of EPN include elevated glucose levels, presence of gasforming organisms, weakened host immunity and impaired tissue perfusion. Escherichia coli (69\%) and Klebsiella pneumoniae (29\%) are noted to be the most common pathogens. Other microbes cultured include Proteus mirabilis, Pseudomonas aeruginosa and Enterococcus species. Anerobes such as Bacteroides fragilis and Clostridium septicum have also been implicated in emphysematous pyelonephritis (Lu et al., 2014). Organisms found in blood cultures were also simultaneously noted in the abscess and urine cultures. High glucose levels may provide a more favorable environment for these organisms, leading to increased rate of growth and catabolism. Enterobacteriaceae lead to mixed acid fermentation of glucose, thus producing gas in the renal parenchyma (Huang and Tseng, 2000). Pathological analysis of gas has shown that they contained hydrogen, carbon dioxide and nitrogen (Huang and Tseng, 2000).

Patients often present with fevers, chills, vague abdominal pain, nausea, flank pain and dysuriasymptoms typical of acute severe pyelonephritis. However, some patients may initially present with severe sepsis, acute kidney injury, thrombocytopenia, altered consciousness and even shock. The left kidney was found to be more frequently involved than the right (67\% Vs 25\%) (Huang and Tseng, 2000; Falagas et al.,
2007). Our patient did not have the typical symptoms of pyelonephritis such as dysuria or flank pain, but was noted to have non-specific lower back tenderness which prompted consideration of pyelonephritis and further imaging. Our clinical suspicion for severe pyelonephritis was high given her history of uncontrolled diabetes. The diagnosis of EPN should be considered in diabetic patients, especially those with uncontrolled or new-onset diabetes and those presenting with symptoms of urinary tract obstruction.

Computed tomography scan is the most reliable diagnostic imaging modality and can be used to characterize the extent of disease (Huang and Tseng, 2000). Ultrasound is operator dependent and not sensitive when imaging gas (Rauf et al., 2007). Gas may appear as nonspecific echogenic foci and may be mistaken for nephrolithiasis. Abdominal x-ray can be utilized as an initial imaging modality, however gas may be seen in only $33 \%$ of cases and may not be distinguishable from air in the bowel (Huang and Tseng, 2000). Likewise, our patient initially had a renal ultrasound. No hydronephrosis was noted, however a large complex mass suspected to be an abscess was seen, along with pyelocalciectasis and questionable left renal calculi; no gas was reported. Given the high clinical suspicion for complicated pyelonephritis, a CT abdomen was ordered, which confirmed the diagnosis of EPN. Of note, the echogenic foci thought to be calculi on ultrasound were confirmed to be areas of gas on CT. Thus, our case further supports the superiority of CT scan in the diagnosis of EPN.

Poor prognostic factors in EPN include thrombocytopenia, azotemia, high urinary red blood cell counts, altered mentation, low blood pressure, worsening proteinuria, shock, altered mental status, extension of infection to the perinephric space and the need for hemodialysis (Aswathaman et al., 2008; Falagas et al., 2007; Misgar et al., 2015). In some cases, the degree of hematuria may be used to identify the extent of necrosis from the infection and the presence of renal venin thrombosis (Misgar et al., 2015). Thrombocytopenia was likely due to severe sepsis and disseminated intravascular coagulation. Mortality increased significantly with the presence of more risk factors, from $27 \%$ with one risk factor to $100 \%$ with several. 
Michaeli et al. (1984) originally characterized emphysematous pyelonephritis into three classes (Sharma et al., 2013). This categorization was later reclassified with the use of CT imaging. Class I is defined as gas in the collecting system; Class II as parenchymal gas only; Class III is further sub-divided into either extension into the perinephric space (Class IIIA) or into the pararenal space (Class IIIB) and Class IV involves bilateral disease (Huang and Tseng, 2000).

There is no consensus on what constitutes ideal management of patients with emphysematous pyelonephritis. However, all patients should be treated with parenteral antibiotics. In patients with Class I disease, antibiotics alone are often sufficient. Patients with Class I and II disease are treated with antibiotics and percutaneous drainage. In patients with Class IV disease (bilateral EPN), nephrectomy is to be avoided and used as a last resort. Antibiotics are chosen by the same guidelines as those for patients with pyelonephritis: A third generation cephalosporin or fluoroquinolone for mild to moderate pyelonephritis (aztreonam for those with a beta lactam allergy) and broader antibiotic coverage (ampicillin-sulbactam, ticeracillin-clavulanate, piperacillin-tazobactam, meropenem, or imipenem) for those patients with severe pyelonephritis, immunosuppression and/or incomplete urinary drainage (Stamm and Hooton, 1993). Vancomycin in combination with imipenem or ceftazidime is the empiric treatment of choice in patients presenting with sepsis and septic shock $\mathrm{Lu}$ et al. (2014). Older patients with uncontrolled diabetes presenting with symptoms of septic shock with a prior history of recently treated pyelonephritis should undergo emergent nonenhanced computed tomography scan (Chen and Chen, 2014).

For patients with IIIA or IIIB classification and two or more prognostic factors (thrombocytopenia, shock, altered sensorium and need for hemodialysis), nephrectomy may be considered. Nephrectomy may be necessary in all patients who fail therapy with antibiotics and percutaneous drainage (Aswathaman et al., 2008; Falagas et al., 2007). Patients with severe hypoalbuminemia were found to have a higher risk of conservative treatment failure (Falagas et al., 2007). However, more recent studies show that the treatment success rate was over $80 \%$ with a combination of antibiotics and percutaneous drainage. Treatment has evolved over the years from a surgical approach such as emergent nephrectomy, to a more conservative approach including intravenous antibiotics and/or percutaneous drainage (Aswathaman et al., 2008). A systematic review of 210 patient cases noted that mortality was significantly lower in those patients treated with antibiotics and percutaneous drainage as compared to nephrectomy (Somani et al., 2008). Our patient had Class II/III disease with two risk factors: thrombocytopenia and presented with severe sepsis. The diagnosis was made within hours of presentation and she was treated successfully with intravenous antibiotics, percutaneous drainage, strict glucose control and monitored with serial imaging. Early diagnosis is essential, as delays have been associated with increasing complications and higher mortality (Huang and Tseng, 2000). However, a month later she continued to have worsening renal function and underwent left laparoscopic radical nephrectomy.

For patients with EPN, the duration of antibiotic treatment has not been clearly defined. Our patient was continued on parenteral antibiotics for more than two weeks in the hospital, then discharged on intravenous and oral antibiotics for another two weeks. She defervesced after 13 days of treatment but continued to experience occasional chills. A repeat CT showed mild improvement and decrease in the size of the abscesses. After nephrectomy, she was continued on intravenous antibiotics and then transitioned to oral therapy for an additional week on discharge. Some patients remain on antibiotic therapy after intervention and the duration reported ranges from 10 days to 30 days depending on severity of the illness (Arsene et al., 2014). Oral antibiotics may be continued afterwards for one to three months (Best et al., 1999; Jain et al., 2000; Laway et al., 2012; Tahir et al., 2000). There are currently no established guidelines for recommended duration of antibiotics for EPN.

Early diagnosis of EPN is essential as it carries a high mortality. Patients with uncontrolled diabetes and urinary tract obstruction are particularly at risk. Prompt computed tomography is essential to establish the diagnosis. Early resuscitation, broad antimicrobial therapy and percutaneous drainage are the mainstays of initial therapy. A strong clinical suspicion is required, as EPN may have an atypical presentation (Ali and Barlas, 2010) and may masquerade as an ordinary urinary tract infection.

\section{Acknowledgment}

The authors declare no conflict of interest. No funding was received.

\section{Author's Contributions}

Tasnim F. Imran: Concept data acquisition, literature search, composing manuscript, selecting images, revision.

Frederick Yick: Literature search, composing, manuscript, editing of images, table.

Ahmed H. Qavi: Literature search, manuscript, editing images.

Sundar Dhamotharan: Critical revision of manuscript.

Steven J. Sperber: Editing, composition, critical revision of manuscript.

\section{Ethics}

Patient consent was obtained prior to publication. 


\section{References}

Kelly, H.A. and W.G. Maccallum, 1898. Pneumaturia. J. Am. Med. Assoc., 31: 375-81. DOI: $10.1001 /$ jama.1898.92450080001001

Aswathaman, K., G. Gopalakrishnan, L. Gnanaraj, N.K. Chacko and N.S. Kekre et al., 2008. Emphysematous pyelonephritis: Outcome of conservative management. Urology, 71: 1007-9. DOI: 10.1016/j.urology.2007.12.095

Best, C.D., M.K. Terris, J.R. Tacker and J.H. Reese, 1999. Clinical and radiological findings in patients with gas forming renal abscess treated conservatively. J. Urol., 162: 1273-6. DOI: 10.1016/S0022-5347(05)68263-8

Yao, J., O.M. Gutierrez and J. Reiser, 2007. Emphysematous pyelonephritis. Kidney Int., 71: 462-5. DOI: $10.1038 /$ sj.ki.5002001

Ali, M. and N.B. Barlas, 2010. Emphysematous pyelonephritis: A case report. Int. J. Diabetes Mellitus, 2: 130-2.

Mokabberi, R. and K. Ravakhah, 2007. Emphysematous urinary tract infections: Diagnosis, treatment and survival (case review series). Am. J. Med. Sci., 333: 111-6.

Lu, Y.C., B.J. Chiang, Y.H. Pong, K.H. Huang and P.R. Hsueh et al., 2014. Predictors of failure of conservative treatment among patients with emphysematous pyelonephritis. BioMed Central Infect. Dis., 14: 418-418.

DOI: $10.1186 / 1471-2334-14-418$

Huang, J.J. and C.C. Tseng, 2000. Emphysematous pyelonephritis: Clinicoradiological classification, management, prognosis and pathogenesis. Archives Internal Med., 160: 797-805.

DOI: $10.1001 /$ archinte.160.6.797

Falagas, M.E., V.G. Alexiou, K.P. Giannopoulou and I.I. Siempos, 2007. Risk factors for mortality in patients with emphysematous pyelonephritis: A metaanalysis. J. Urol., 178: 880-5. PMID: 17631348

Rauf, A.A., A. Shanaah, A. Joshi, S. Popli and M. Vaseemuddin et al., 2007. Failure of sonography to visualize a kidney affected by emphysematous pyelonephritis. Indian J. Urol. Society, 23: 200-2. DOI: $10.4103 / 0970-1591.32077$
Misgar, R.A., A.I. Wani, M.I. Bashir, N.A. Pala and I. Mubarik et al., 2015. Successful medical management of severe bilateral emphysematous pyelonephritis: Case studies. Clinical Diabetes, 33: 76-9. DOI: $10.2337 /$ diaclin.33.2.76

Michaeli, J., P. Mogle, S. Perlberg, S. Heiman and M. Caine, 1984. Emphysematous pyelonephritis. J. Urol., 131: 203-8. DOI: 10.1056/NEJMicm1314314

Sharma, P.K., R. Sharma, M.K. Vijay, P. Tiwari and A. Goel et al., 2013. Emphysematous pyelonephritis: Our experience with conservative management in 14 cases. Urol. Annals, 5: 157-62. DOI: $10.4103 / 0974-7796.115734$

Stamm, W.E. and T.M. Hooton, 1993. Management of urinary tract infections in adults. New England J. Med., 329: 1328-34. DOI: 10.1056/NEJM199310283291808

Chen, C.Y. and C.J. Chen, 2014. Images in clinical medicine. Emphysematous pyelonephritis. N. Engl. J. Med., 371: e34- e34. DOI: 10.1056/NEJMicm1314314

Somani, B.K., G. Nabi, P. Thorpe, J. Hussey and J. Cook et al., 2008. Is percutaneous drainage the new gold standard in the management of emphysematous pyelonephritis? Evidence from a systematic review. J. Urol., 179: 1844-9. DOI: 10.1016/j.juro.2008.01.019

Arsene, C., A. Saste, S. Arul, J. Mestrovich and R. Kammo et al., 2014. A case series of emphysematous pyelonephritis. Case Reports Med. DOI: $10.1155 / 2014 / 587926$

Jain, S.K., N. Agarwal and S.K. Chaturvedi, 2000. Emphysematous pyelonephritis: A rare presentation. J. Postgrad. Med., 46: 31-32. PMID: 10855076

Laway, B.A., M.A. Bhat, M.I. Bashir, M.A. Ganie and S.A. Mir et al., 2012. Conservative management of emphysematous pyelonephritis. Indian J. Endocrinol. Metabolism, 16: 303-305. DOI: $10.4103 / 2230-8210.93776$

Tahir, H., G. Thomas, N. Sheerin, H. Bettington and J.M. Pattison et al., 2000. Successful medical treatment of acute bilateral emphysematous pyelonephritis. Am. J. Kidney Dis., 36: 1267-70. DOI: 10.1053 /ajkd.2000.19844 